\title{
Overexpression of Endoplasmic Reticulum Oxidoreductin 1- $\alpha$ (ER01L) Is Associated with Poor Prognosis of Gastric Cancer
}

\author{
So-Young Seol, MS ${ }^{1}$ \\ Chul Kim, MD, PhD² \\ Jae Yun Lim, MD, PhD' \\ Sun Och Yoon, MD, PhD ${ }^{3}$ \\ Soon Won Hong, MD, PhD 3 \\ Jong Won Kim, MD, PhD ${ }^{4}$ \\ Seung Ho Choi, MD, PhD 4 \\ Jae Yong Cho, MD, $\mathrm{PhD}{ }^{1}$
}

\begin{abstract}
${ }^{1}$ Department of Medical Oncology, Gangnam Severance Cancer Hospital, Yonsei University College of Medicine, Seoul, ${ }^{2}$ Samsung Bioepis Co., Ltd., Incheon, Departments of ${ }^{3}$ Pathology and ${ }^{4}$ Surgery, Gangnam Severance Cancer Hospital, Yonsei University College of Medicine, Seoul, Korea
\end{abstract}

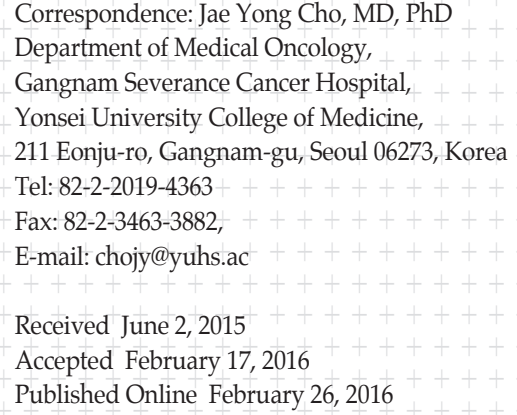

\begin{abstract}
Purpose
Gastric cancer is the second leading cause of cancer-related death worldwide. Although surgery is the standard curative treatment for gastric cancer, relapse occurs in a large number of patients, except in the case of early diagnosed gastric cancer. Following previous studies that identified endoplasmic reticulum oxidoreductin 1- $\alpha$ (ERO1L) as a potential marker for gastric cancer, we investigated the functional role of ERO1L in gastric cancer.
\end{abstract}

\section{Materials and Methods}

For validation of microarray data, the mRNA expression level of ER01L was measured by quantitative real-time reverse transcription polymerase chain reaction in 56 independent stage III gastric cancer patients. Immunohistochemical staining was performed to examine the protein expression level of ERO1L in 231 gastric cancer patients. Correlation between gene expression and cancer prognosis was evaluated.

\section{Results}

Patients with high ER01L expression had poorer survival than those with low expression $(p<0.01)$. Functional assays demonstrated that ER01L knockdown inhibited cell proliferation, migration, invasion, and chemoresistance. In addition, involvement of inactivation of Akt and JNK signaling in molecular mechanisms of ERO1L inhibition was demonstrated.

\section{Conclusion}

High expression of ERO1L is associated with poor prognosis of patients with gastric cancer. These results indicate that ERO1L expression may be a clinically promising therapeutic target for prevention of gastric cancer.

\section{Introduction}

Gastric cancer is the second leading cause of cancer-related death worldwide [1]. Despite a decrease in the incidence of gastric cancer in some developed countries over the past decade, it is estimated that one million new cases are diagnosed each year. The clinical diversity of gastric cancer arises from its diverse molecular biology [2,3], caused by changes in the expression of various genes. Current chemotherapy-

\section{Key words}

ER01L, Stomach neoplasms, Prognosis,

Molecular targeted therapy based treatments for advanced gastric cancer do not demonstrate a substantial effect on overall survival (OS) [4,5]. Thus, identification of new, effective therapeutic approaches is of great urgency [6-12].

Hypoxia, a state of reduced oxygen availability, is an important factor making tumors less responsive to radiation and chemotherapy. Numerous studies have shown that hypoxic tumors have a more aggressive phenotype [13], thus, tumor hypoxia correlates with a more aggressive disease course and limits the effectiveness of anti-cancer therapies. 
Endoplasmic reticulum oxidoreductin 1- $\alpha$ (ERO1L) is a hypoxia-inducible endoplasmic reticulum-resident oxidase that regulates the post-translational branch of oxidized protein folding $[14,15]$. Human ERO1L is expressed at a very low level in normoxic cells. However, it has been shown that ERO1L is induced in hypoxic cells in response to low oxygen availability [15]. Interestingly, co-induction of ERO1L and vascular endothelial growth factor (VEGF) in hypoxic tumor cells suggests that ERO1L might be harnessed for reducing levels of this key pro-angiogenic factor. Considering that hypoxia-induced secretion of VEGF plays a key role in tumor angiogenesis, findings reported here suggest ERO1L as a potential anti-angiogenic target [16].

In this study, we further investigated the clinical significance and functional role of ERO1L expression in gastric cancer. First, analysis of ERO1L expression in gastric cancer was performed using quantitative real-time reverse transcription polymerase chain reaction (qRT-PCR) and immunohistochemical staining in gastric cancer patients. We also identified the relationship between ERO1L expression and clinicopathological features and evaluated its prognostic value in post-resection survival of gastric cancer patients. In addition, we evaluated the functional role of ERO1L in gastric cancer by examining the proliferation, migration, and invasion of gastric cell lines.

\section{Materials and Methods}

\section{Patients and samples}

Tumor specimens and clinical data were obtained from patients with primary gastric adenocarcinoma who underwent curative gastrectomy as the primary treatment between 1999 and 2007 at Yonsei University, Severance Hospital (Seoul, Korea), and Yonsei University, Gangnam Severance Hospital (Seoul, Korea). Three patient groups were used for each experiment: group 1 (65 gastric cancer patients underwent curative surgery as a primary treatment) for microarray; group 2 (56 formalin-fixed, paraffin-embedded tissue from a separate gastric adenocarcinoma patient group) for validation tests using qRT-PCR; and group 3 (paraffinembedded samples from 231 patients with gastric cancer) for tissue microarray immunohistochemistry. All samples were collected after obtaining written informed consent from patients, and the study was approved by the Institutional Review Board of Yonsei University, Gangnam Severance Hospital (Seoul, Korea).

\section{Gene expression microarray data analysis}

The previously generated gene expression data from gastric cancer patients are available in NCBI's GEO public database (microarray data accession number, GSE13861) [12]. Sixty-five gastric cancer patients underwent curative surgery as a primary treatment, with clinical data obtained from Yonsei University, Severance Hospital (Table 1). Sixty-five surgically removed frozen gastric adenocarcinoma tissues and 19 normal surrounding tissue samples were used for the microarray experiments. Total RNA was extracted using a mirVana RNA Isolation Labeling Kit (Ambion, Austin, TX). For labeling and hybridization, $500 \mathrm{ng}$ of total RNA was used, according to the manufacturer's protocols (HumanHT12 v.3 Expression BeadChip, Illumina, San Diego, CA). The microarray data were normalized using the quantile normalization method in the Linear Models for Microarray Data (LIMMA) package in the $\mathrm{R}$ language environment [17]. The expression level of each gene was transformed into a log2 base before additional analysis was performed. The random variance $t$ test was applied for identification of differentially expressed genes between the two tissue types. The differences in gene expression were considered significant if the p-value was less than 0.001 . Cluster analysis was performed using Cluster 3.0 and TreeView24 [18]. Univariate analysis was performed by dividing the patients into two groups based on the median value of each gene expression level to search for prognostic genes.

\section{3. qRT-PCR and analysis}

Stage III samples were chosen from 56 different gastric cancer patients for validation of the microarray data. Total RNA was extracted according to the manufacturer's instruction manual (RecoverAll Total Nucleic Acid Isolation, Ambion). ERO1L gene expression was assayed using qRT-PCR with specific Taqman primers (Applied Biosystems, Foster City, CA). Real-time reverse transcription polymerase chain reaction (PCR) amplification was performed using the 7900HT Fast Real-Time PCR System with a 384-well block module (Applied Biosystems). Cycling conditions were $48^{\circ} \mathrm{C}$ for 30 minutes and $95^{\circ} \mathrm{C}$ for 10 minutes, followed by 40 cycles at $95^{\circ} \mathrm{C}$ for 15 seconds and $60^{\circ} \mathrm{C}$ for 60 seconds. Total RNA was isolated from cultures of gastric cancer cells grown in 6-well plates using TRIzol (Invitrogen, Carlsbad, CA) according to the manufacturer's protocol. cDNA was synthesized from $1 \mu \mathrm{g}$ of total RNA using a Maxima First Strand cDNA Synthesis Kit (Thermo Scientific, Rockford, IL). Real-time quantitative PCR amplifications were performed by SYBR Green assay on a 7500 Real-Time PCR System with 96-well block module (Applied Biosystems). SYBR Green PCR conditions were $50^{\circ} \mathrm{C}$ for 2 minutes and $95^{\circ} \mathrm{C}$ for 10 minutes, followed 
Table 1. Clinicopathological factors of gastric cancer patients

\begin{tabular}{|c|c|c|c|}
\hline Characteristic & Microarray (n=65) & qRT-PCR (n=56) & TMA $(n=231)$ \\
\hline \multicolumn{4}{|l|}{ Age } \\
\hline Mean (range, yr) & $63(32-83)$ & $60(28-76)$ & $58(25-77)$ \\
\hline \multicolumn{4}{|l|}{ Sex } \\
\hline Male & $46(71)$ & $31(55)$ & $144(62)$ \\
\hline Female & $19(29)$ & $25(45)$ & $87(38)$ \\
\hline \multicolumn{4}{|l|}{ Follow-up duration } \\
\hline Median (95\% CI, mo) & $41.7(41-42)$ & 83.8 (78.6-91.4) & $95.9(91.3-97.1)$ \\
\hline \multicolumn{4}{|l|}{ Histological type } \\
\hline Intestinal & $23(35)$ & $15(27)$ & $80(35)$ \\
\hline Diffuse & $42(65)$ & $41(73)$ & $151(65)$ \\
\hline \multicolumn{4}{|l|}{ TNM stage } \\
\hline I & $12(18)$ & 0 & $92(40)$ \\
\hline II & $11(17)$ & 0 & $58(25)$ \\
\hline III & $26(40)$ & $56(100)$ & $64(28)$ \\
\hline IV & $16(25)$ & 0 & $17(7)$ \\
\hline \multicolumn{4}{|l|}{ Location } \\
\hline Cardia & $5(8)$ & $10(18)$ & $20(9)$ \\
\hline Non-cardia & $60(92)$ & $46(82)$ & $211(91)$ \\
\hline \multicolumn{4}{|l|}{ Adjuvant chemotherapy } \\
\hline Yes & $49(75)$ & $55(98)$ & $143(62)$ \\
\hline No & $16(25)$ & $1(2)$ & $88(38)$ \\
\hline
\end{tabular}

Values are presented as number (\%) unless otherwise indicated. qRT-PCR, quantitative real-time reverse transcription polymerase chain reaction; TMA, tissue microarray; $\mathrm{CI}$, confidence interval.

by $95^{\circ} \mathrm{C}$ for 50 seconds, $60^{\circ} \mathrm{C}$ for 50 seconds, and $72^{\circ} \mathrm{C}$ for 1 minute for 40 cycles. SYBR Green Master Mix contains an internal passive dye, ROX, in addition to SYBR Green dye. Relative amounts of mRNA were calculated from the threshold cycle $\left(C_{T}\right)$ number based on expression of $\beta-2$ microglobulin or $\beta$-actin as an endogenous control. All experiments were triplicated and the values averaged.

\section{Tissue microarray construction and immunohistochem- ical staining}

Paraffin-embedded tissue microarray blocks of gastric cancer tissue specimens were created from 231 patients. Each block contained 3-mm cores of gastric cancer tissue. The $4-\mu \mathrm{m}$-thick sections were deparaffinized and processed to block endogenous peroxidase activity. Next, an antigen retrieval step was performed, and primary anti-ERO1L antibody (1:100, monoclonal, Abnova, Taipei, Taiwan) was subsequently applied to the sections. The sections were then incubated with a secondary antibody (HRP-mouse), and the stains were developed using a Nova-RED Substrate Kit (Vector Laboratory, Burlingame, CA). The samples were then counterstained with Harris hematoxylin. ERO1L protein expression levels were evaluated by two pathologists. The staining intensity was recorded as score 0 , negative; score 1 , weaker than normal; score 2, equally intense as normal; and score 3 , strong than normal. High-expression was defined as a staining score $\geq 3$ and low-expression as a staining score $<3$. For slides heterogeneously stained within a tumor, we graded the highest intensity within the tumor.

\section{Cell culture and chemotherapeutic agents}

Gastric cancer cell lines (AGS, SNU1, MKN1, MKN28, MKN45, and NCI-N87) and human embryonic kidney cell lines 293T (HEK 293T) were obtained from low-passage seed stocks at the Korean Cell Line Bank (KCLB). Cells were exposed to hypoxia by placement in a mixed-gas incubator infused with an atmosphere consisting of $94 \% \mathrm{~N}_{2}, 5 \% \mathrm{CO}_{2}$, and $1 \% \mathrm{O}_{2}$. Paclitaxel and 5-fluorouracil (5-FU) were dissolved in dimethylsulfoxide and sterile water, respectively. All chemotherapeutic agents were obtained from SigmaAldrich (St. Louis, MO).

\section{Establishment of stable cell line}

pGIPZ-shERO1L and pGIPZ-shNonTarget vectors were purchased from Dharmacon (Dharmacon, Chicago, IL). 

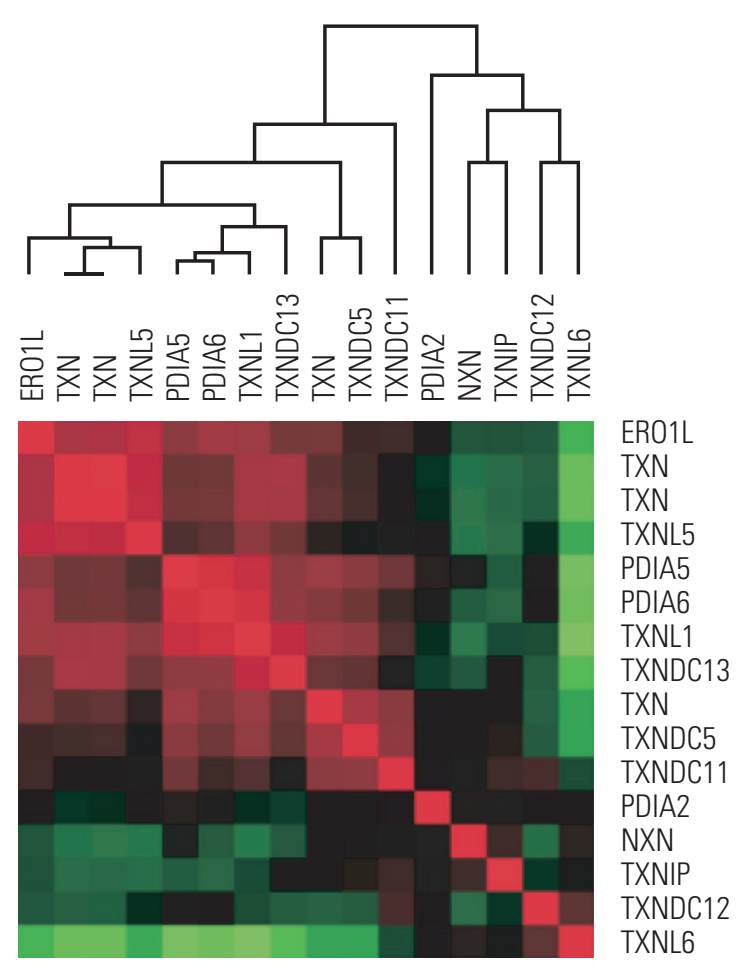

Fig. 1. ERO1L and TXN family in-trans correlation in human gastric cancer. Data are given in NCBI's GEO public database (microarray data accession number, GSE13861). The color red or green reflects relative high or low expression level, respectively. ERO1L, endoplasmic reticulum oxidoreduction $1-\alpha$; TXN, thioredoxin.

pGIPZ-shERO1L and pGIPZ-shNonTarget lentiviral vectors were transfected into HEK 293T cells in 60-mm dishes using Fugene 6 (Promega, Madison, WI) according to the manufacturer's protocol. Culture medium containing virus particles was collected 48 hours later and added to gastric cancer cell lines. Twenty-four hours later, transduced cells were selected for 10 days with puromycin (Invitrogen).

\section{Boyden chamber assay}

After trypsinization, invasiveness of cells was tested in vitro by Boyden chamber invasion assay (Neuro Probe 48-well Micro Chemotaxis Chamber, Neuro Probe Inc., Gaithersburg, MD). Matrigel (BD Transduction Lab, San Jose, CA) was diluted to $25 \mathrm{mg} / 50 \mu \mathrm{L}$ with cold distilled water and then applied to 8 - $\mu \mathrm{m}$ pore size polycarbonate membrane filters. Cells were seeded in the upper part of the Boyden chamber at a density of $1 \times 10^{5}$ cells / well in $50 \mu \mathrm{L}$ of serum free medium and then incubated for 24 hours at $37^{\circ} \mathrm{C}$.

The lower chamber also contained standard medium with

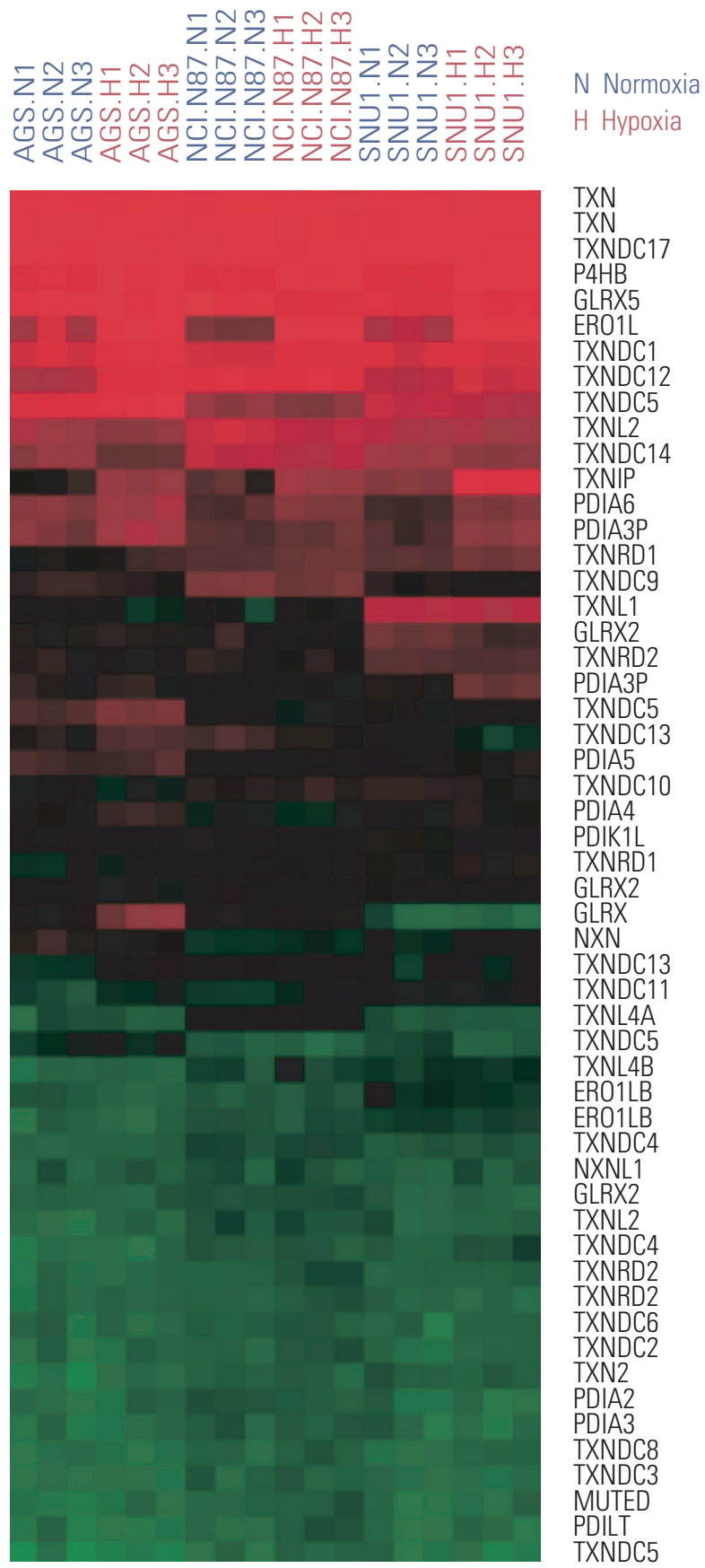

Fig. 2. Two different conditions of gastric cancer cell lines, normoxia and hypoxia, show mRNA expression patterns. mRNA expression patterns of gastric cancer cells (AGS, NCI-N87, and SNU1) in normoxia and hypoxia are depicted in a heat map. The color red or green reflects relative high or low expression level, respectively. 
Table 2. Protein expression of ERO1L protein in gastric cancers

\begin{tabular}{lccc} 
Characteristic & ERO1L high-expression & ERO1L low-expression & p-value \\
Total $(\mathrm{n}=\mathbf{2 3 1})$ & $107(46)$ & $124(54)$ & \\
Histological type & & & \\
\hline Intestinal type $(\mathrm{n}=80)$ & $42(53)$ & $38(47)$ & \\
$\quad$ Diffuse type $(\mathrm{n}=151)$ & $65(43)$ & $86(57)$ & 0.170 \\
Stage & $63(42)$ & $87(58)$ & \\
$\quad$ Stage I/II $(\mathrm{n}=150)$ & $44(54)$ & $37(46)$ & \\
\hline Stage III/IV $(\mathrm{n}=81)$ &
\end{tabular}

Values are presented as number (\%). ERO1L, endoplasmic reticulum oxidoreduction 1- $\alpha$.

$0.1 \%$ fetal bovine serum. Cells that invaded the lower surface of the membranes were stained using a Diff-Quik Stain Kit (Triangle Biochemical Sciences Inc., Durham, NC). Invading cells in five randomly selected fields were counted using Microscope Axio Imager M2 (Carl Zeiss, Thornwood, NY). To determine the effect of ERO1L on cell migration, cells were seeded in a Boyden chamber on membrane filters not coated with Matrigel. Migration of cells was measured as described in the invasion assay.

\section{Cell viability assay and clonogenicity}

Cell viability was determined using an EZ-Cytox Cell Viability Assay Kit (Daeil Lab Service Co., Seoul, Korea) according to the manufacturer's instructions. Briefly, $20 \mu \mathrm{L}$ of EZ-Cytox kit reagent was added to each well of a 96-well microplate, followed by incubation at $37^{\circ} \mathrm{C}$ in a humidified $\mathrm{CO}_{2}$ incubator for 2 hours. Optical density was then measured at a wavelength of $450 \mathrm{~nm}$ using a 96-well microplate reader (Bio-Rad Laboratories Inc., Hercules, CA). The experiment was performed in triplicate.

Cells were harvested and resuspended in complete growth media. Cells were seeded onto 6-well plates $\left(1 \times 10^{3}\right.$ cells / well) and allowed to grow until visible colonies formed (21 days). Cell colonies were fixed with 10\% methanol and 10\% acetic acid, stained with $0.25 \%$ crystal violet in $25 \%$ methanol, washed, and air-dried.

\section{Immunoblotting}

Whole cell extraction was performed using RIPA buffer supplemented with protease inhibitors.

Samples were denatured in sodium dodecyl sulfate (SDS) sample buffer. Total proteins were separated by loading 20 $\mu \mathrm{g}$ of total cell lysate onto a denaturing SDS-polyacrylamide gel and transferred onto a polyvinylidene difluoride membrane. Membranes were blocked with 3\% non-fat dry milk in phosphate-buffered saline containing $0.1 \%$ Triton X-100, followed by incubation with primary antibodies that recognize anti-Ero11 (Santa Cruz Biotechnology, Dallas, TX), antiAkt, anti-phospho-Akt (Ser473), anti-JNK, anti-phospho-JNK (Thr183/Tyr185), anti-glyceraldehyde 3-phosphate dehydrogenase, and actin (Cell Signaling, Danvers, MD). Secondary antibody conjugated to horseradish peroxidase (Vector Laboratories Inc.) was used for detection of primary antibodies, and enzymatic signals were visualized using a chemiluminescence kit (Thermo Scientific). Western blots were quantitated using ImageJ software.

\section{Statistical analysis for survival data}

The BRB-Array Tools system was used for analysis of the microarray data. Statistical analyses were primarily performed using PASW ver. 17.0 (SSPS Inc., Chicago, IL), and graphical interpretations were generated with GraphPad Prism5 (GraphPad Software, San Diego, CA). Differences between groups were analyzed using $t$ test and chi-square test, and Kaplan-Meier plots and log-rank test were used for estimation of patient survival. A p-value of less than 0.05 was considered statistically significant, and all tests were twotailed.

\section{Results}

\section{Prognostic significance of ERO1L expression in gastric cancer patients}

In a previous study, we reported on the important role of thioredoxin (TXN) in gastric cancer. Approximately 7,000 genes were significantly correlated with TXN according to the bioinformatics in-trans correlation analysis of TXN expression using whole mRNA-expressing genes $(p<0.001)$. In-trans correlation analysis between TXN family genes 

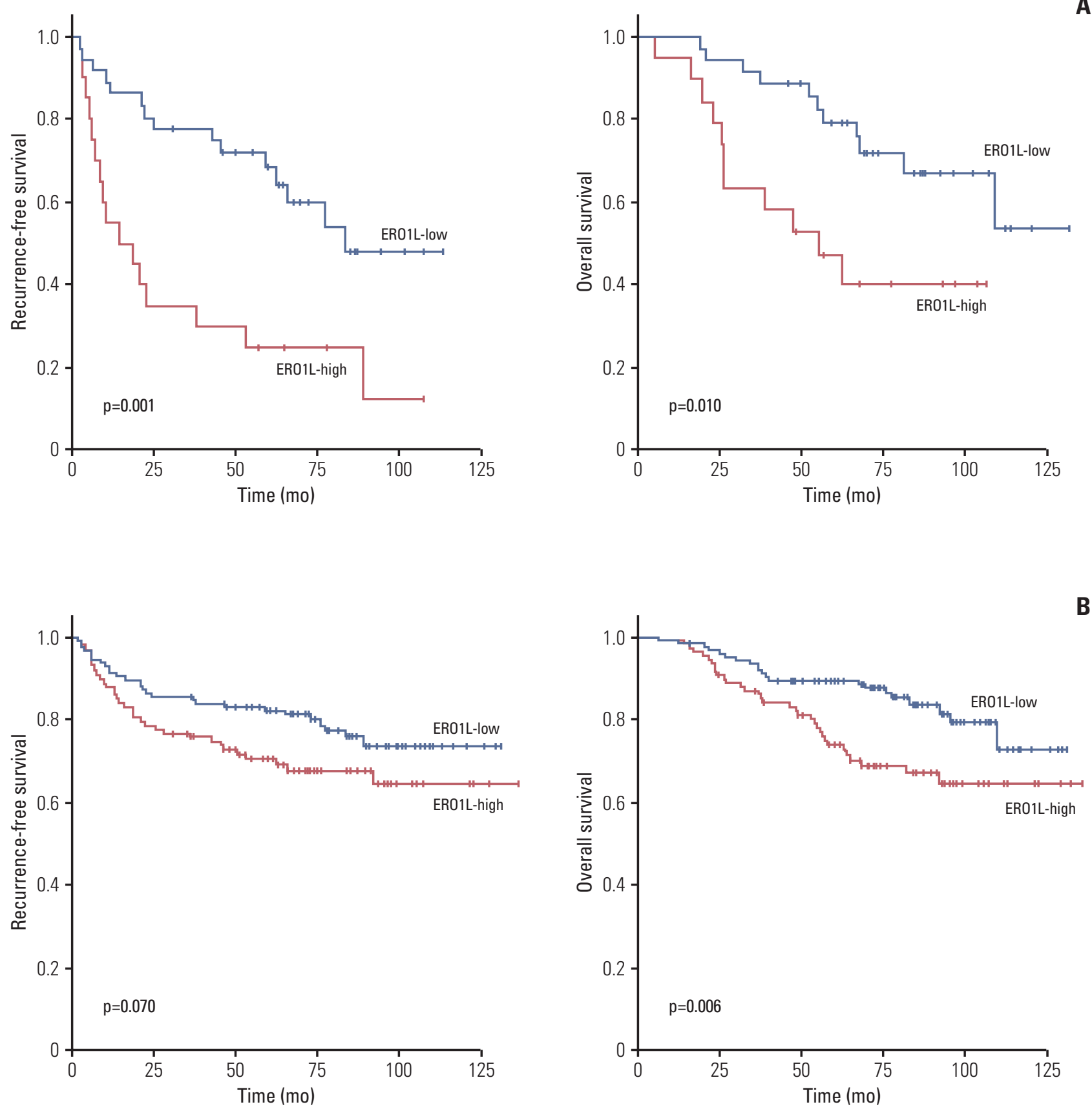

Fig. 3. Kaplan-Meier plot of recurrence-free survival (RFS) and overall survival (OS) according to gene expression. (A) Gene expression was measured using quantitative reverse transcription polymerase chain reaction in 56 stage III gastric cancer patients. (B) Gene expression was measured using immunohistochemical staining analysis in 231 gastric cancer patients divided into two groups according to their gene expression levels. ERO1L, endoplasmic reticulum oxidoreduction 1- $\alpha$. 


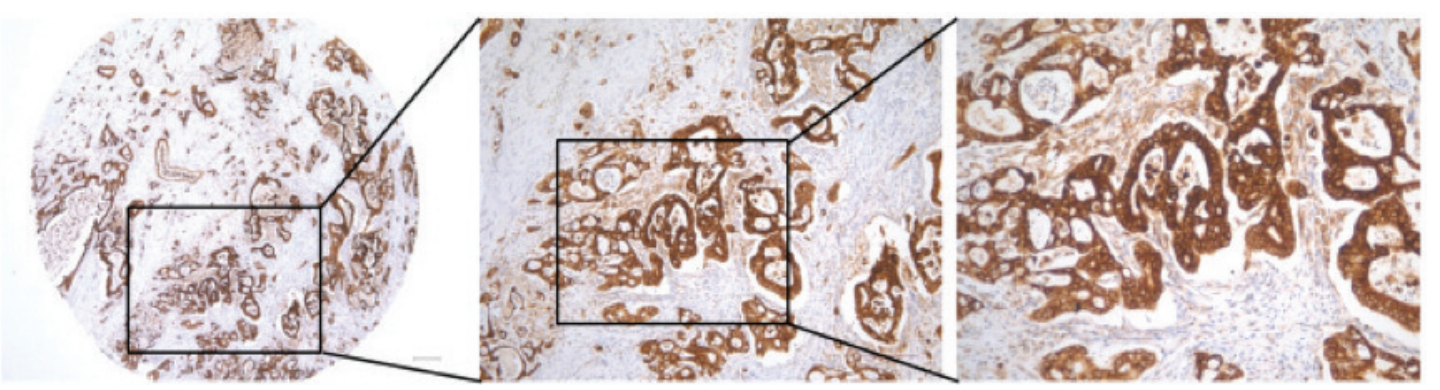

Fig. 4. Representative images of endoplasmic reticulum oxidoreduction 1- $\alpha$ (ERO1L) protein expression in gastric cancer. High immunohistochemical staining of ERO1L in cytoplasm of gastric cancer (left, $\times 40$; middle, $\times 100$; right, $\times 200$ ).

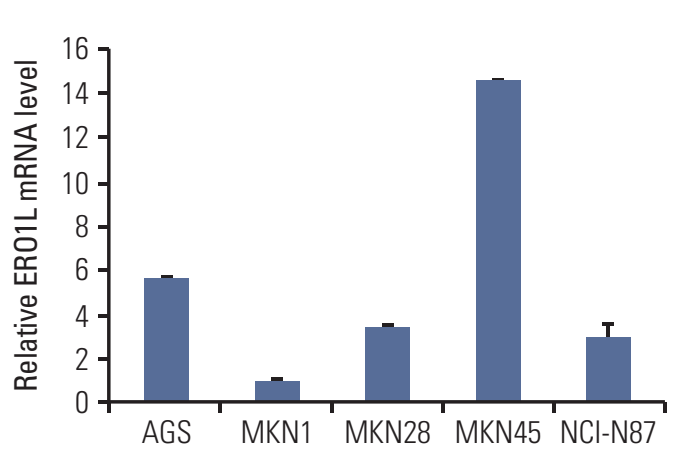

B

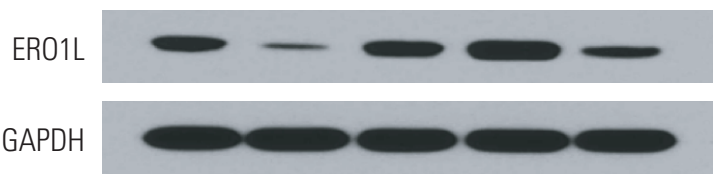

Fig. 5. Endoplasmic reticulum oxidoreduction 1- $\alpha$ (ERO1L) expression in gastric cancer cell lines. (A) ERO1L mRNA levels were assessed using quantitative real-time reverse transcription polymerase chain reaction. Expression of $\beta$-actin was included as an internal loading control. (B) ERO1L protein levels were analyzed using immunoblotting. Glyceraldehyde 3-phosphate dehydrogenase (GAPDH) was included as an internal loading control.

showed positive correlation of ERO1L with TXN ( $\mathrm{r}=0.586$, $\mathrm{p}<0.001$ ) (Fig. 1), suggesting that tumors were in a relatively high hypoxic state. ERO1L and TXN family member genes were highly expressed under hypoxic conditions in gastric cancer cell lines (AGS, SNU1, and NCI-N87) (Fig. 2).

To determine the association between $E R O 1 L$ gene expression and prognosis among patients with the same stage of cancer, 56 stage III patients were randomly selected for qRT-
PCR analysis (Table 1). The median follow-up duration after curative resection was 83.8 months. By the last follow-up visit, 31 patients had experienced recurrence, and 22 patients had died of gastric cancer. The patients were divided into high or low expression groups according to ERO1L expression level. Recurrence-free survival (RFS) and OS were analyzed between the two groups. Expression of ERO1L in stage III gastric cancer patients showed a significant association with RFS ( $\mathrm{p}=0.001)$ and OS ( $\mathrm{p}=0.010$ ) (Fig. 3A).

To examine the relationship between ERO1L expression and clinicopathological features, immunohistochemical assay analysis of ERO1L was performed in 231 gastric cancer specimens. ERO1L was overexpressed in approximately $46 \%$ of cancer tissues regardless of histological type and TNM stage (Table 2). There was no association between histological cell type or tumor stage and ERO1L expression level ( $\mathrm{p}=0.170$ or $\mathrm{p}=0.073$, respectively). Representative immunohistochemical staining results are shown in Fig. 4. RFS and OS were analyzed between the two groups (ERO1L-high and ERO1L-low), as shown in the immunohistochemical staining results (Fig. 3B). ERO1L protein expression influenced OS $(\mathrm{p}=0.006)$, whereas ERO1L protein expression did not show a significant association with RFS ( $\mathrm{p}=0.070)$. Taken together, these data indicate that high ERO1L expression was predictive of a poor prognosis for gastric cancer patients.

\section{ERO1L expressed in human gastric cancer cell lines}

We next evaluated whether or not human gastric cancer cell lines express ERO1L and thus their utility in assessing the potential function of ERO1L protein. The mRNA expression level of ERO1L was measured by qRT-PCR analysis (Fig. 5A), and Western blots analysis confirmed EOR1L protein expression in gastric cancer cell lines (Fig. 5B). Strong EOR1L expression was observed in primary and metastatic gastric carcinoma cells (AGS, established from a primary tumor; MKN45, metastatic). These results suggest that at 

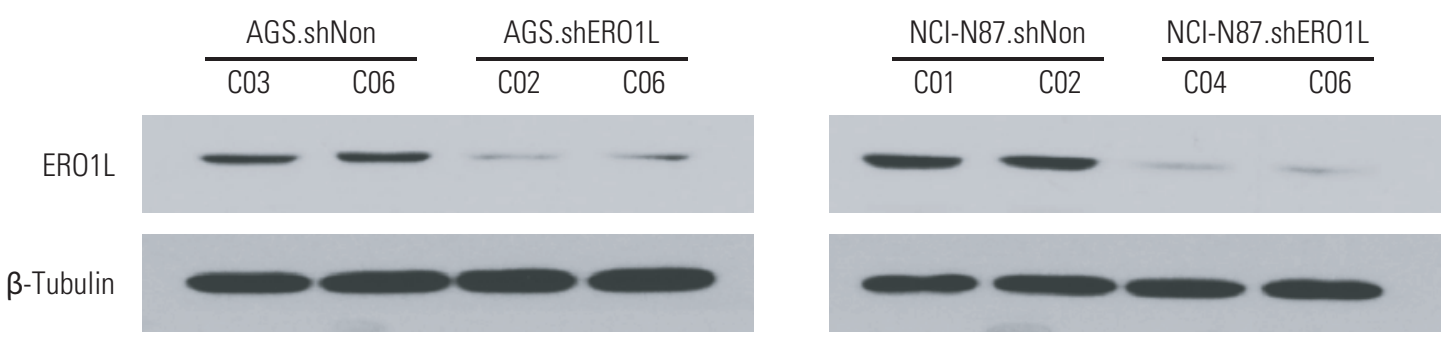

A
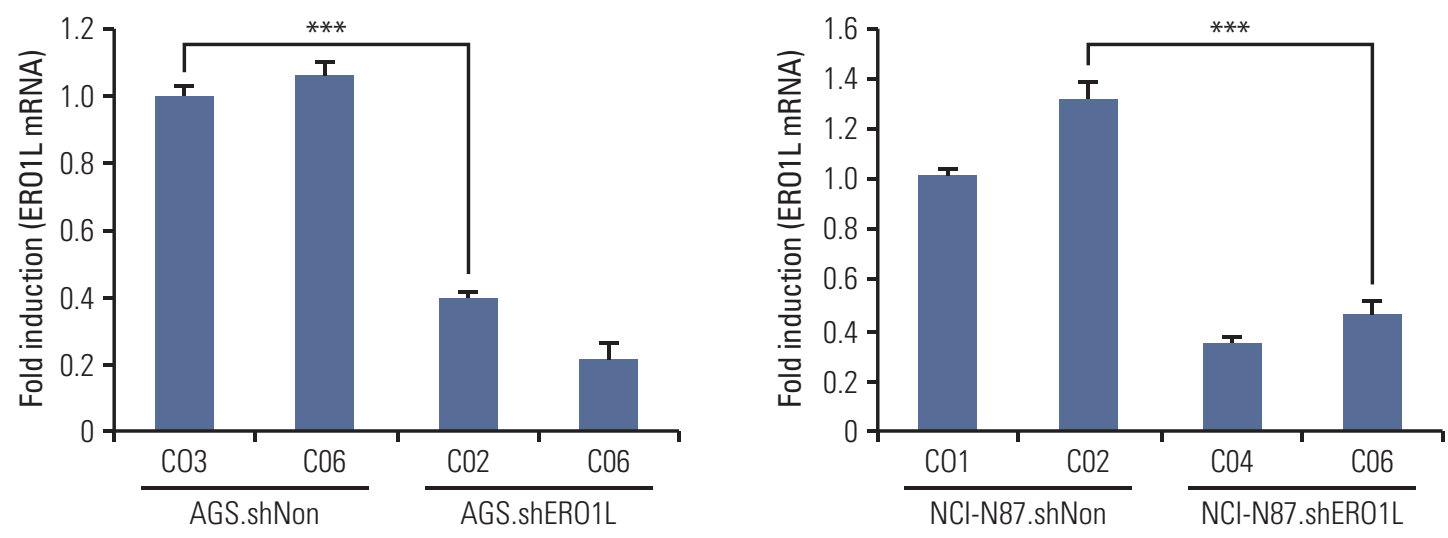

Fig. 6. Endoplasmic reticulum oxidoreduction 1- $\alpha$ (ERO1L) knockdown expression in lentivirus-mediated stable cells. Total RNA and whole cell lysates were collected from AGS and NCI-N87 cells transfected with shNonTarget and shERO1L vector (AGS, shNon.C03 and C06/shERO1L.C02 and C06; NCI-N87, shNon.C01 and C02/shERO1L.C04 and C06). (A) Expression of ERO1L protein was analyzed by immunoblotting, and mRNA level was measured by quantitative real-time reverse transcription polymerase chain reaction. $\beta$-Tubulin and $\beta$-actin were included as an internal loading control. ${ }^{* * *} \mathrm{p}<0.001$.

least a subset of well-credentialed gastric cancer cell lines retains ERO1L expression, thereby allowing for further functional characterization. As a functional study, we selected two gastric cancer cell lines (AGS and NCI-N87) that demonstrated ERO1L expression by qRT-PCR and microarray analyses.

\section{ERO1L knockdown reduces cell proliferation in gastric cancer cells}

The biological function of ERO1L in gastric cancer is still unknown. To examine the potential role of ERO1L in gastric cancer cells, we first knocked down ERO1L in gastric cancer cell lines (AGS and NCI-N87) using ERO1L-specific shRNAs. The two most efficiently knocked down cells for each cell type were selected to further examine phenotypic changes arising from ERO1L expression. Knockdown yielded $>60 \%$ reduction of EOR1L mRNA and protein expression (Fig. 6).

We analyzed the effects of ERO1L-deficient gastric cancer cells (AGS, shERO1L.C02 and C06; NCI-N87, shERO1L.C04 and C06) compared with shNonTarget vector-infected control cells (AGS, shNon.C03 and C06; NCI-N87, shNon.C01 and C02). Proliferation was tested in short-term proliferative impact (WST-1 assay) and long-term proliferative ability (colony formation assay). ERO1L shRNA-mediated knockdown resulted in significantly reduced growth of gastric cancer cell lines $(p<0.001)$ (Fig. 7A). Similar results were also observed for colony formation, which was also significantly inhibited $(\mathrm{p}<0.001)$ in shERO1L cells compared with shNonTarget cells (Fig. 7B).

\section{ERO1L expression is associated with gastric cancer cell invasiveness and migration}

Next, we tested whether or not ERO1L expression is involved in progression of gastric cancer to metastatic disease, especially in late tumorigenesis, including migration and invasion. 

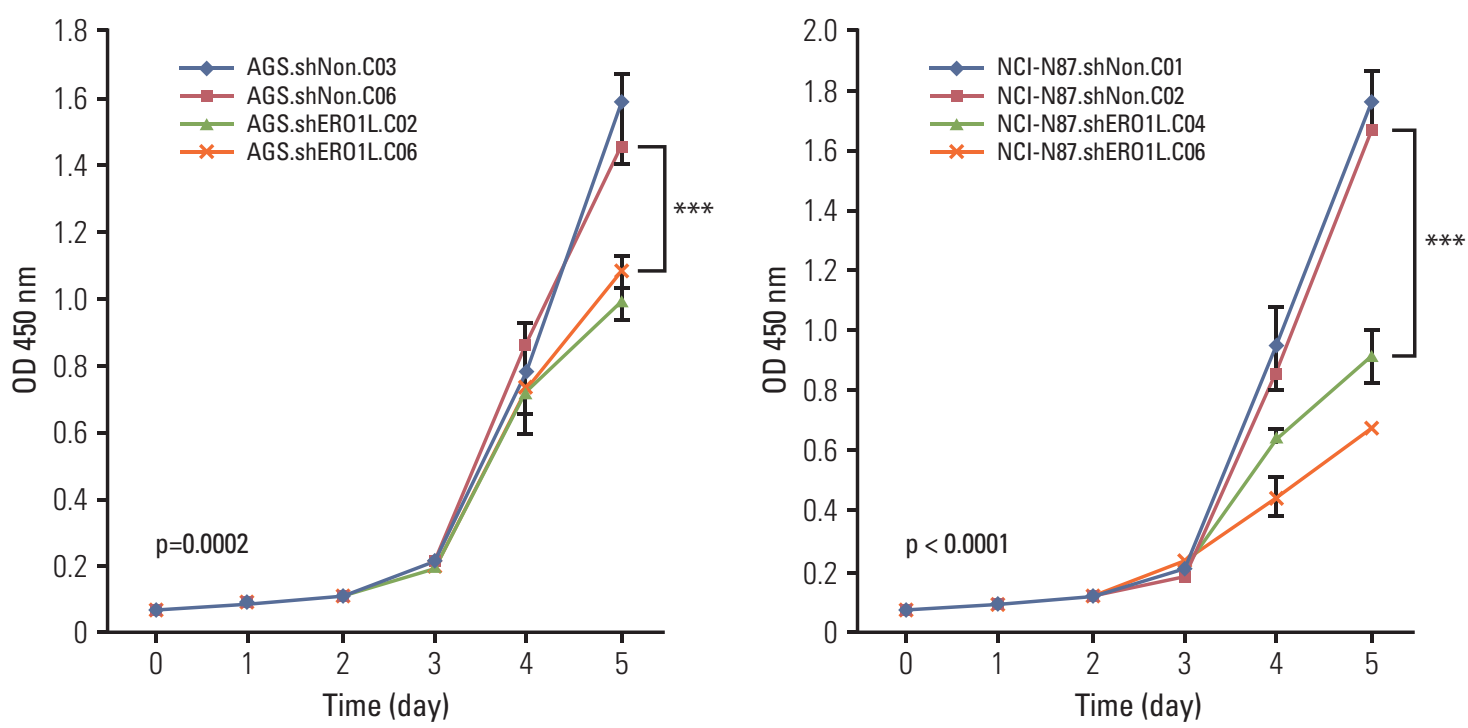

A
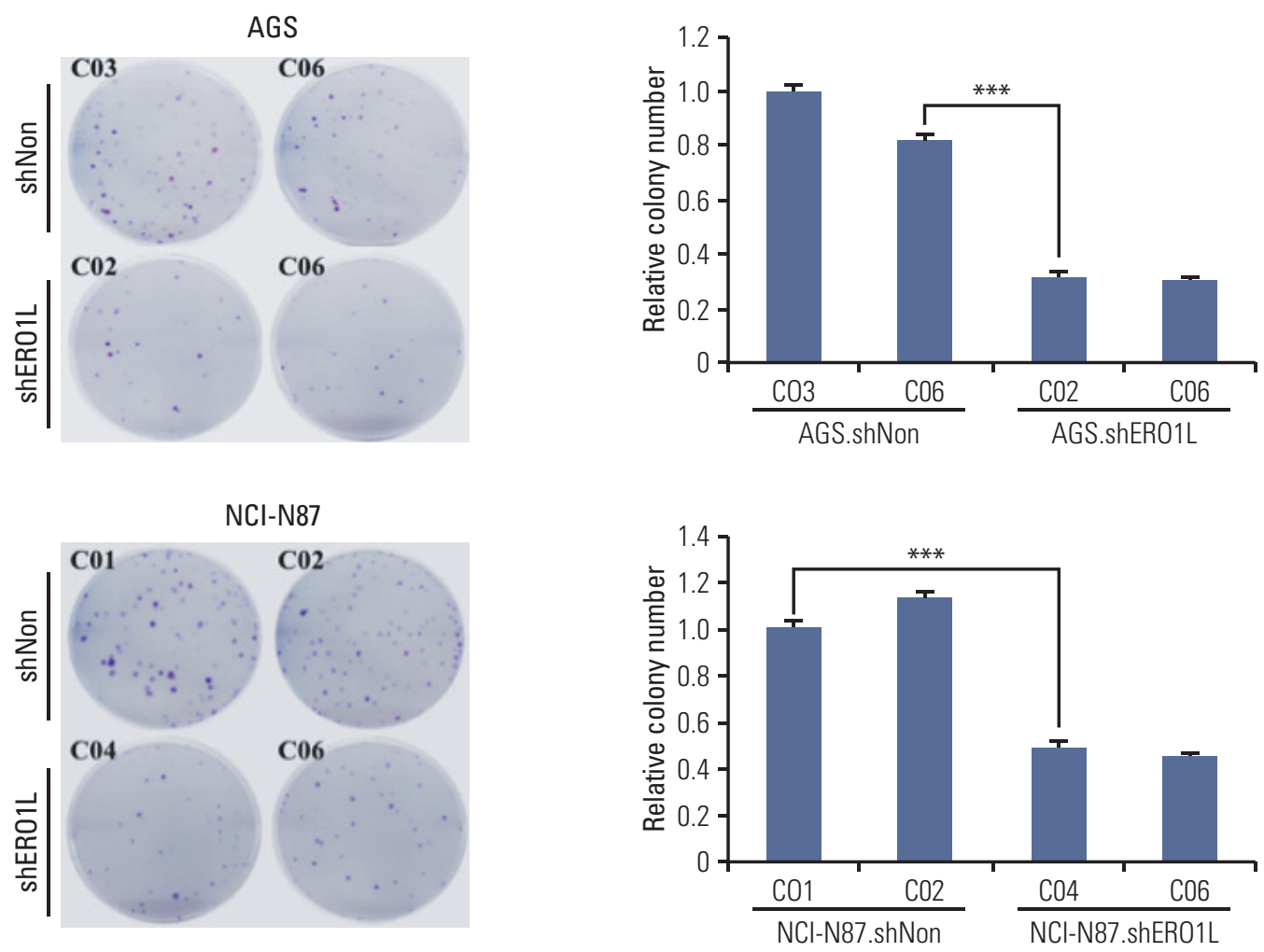

Fig. 7. Endoplasmic reticulum oxidoreduction 1- $\alpha$ (ERO1L)-deficient gastric cancer cells decrease cell proliferation. (A) Cell proliferation was determined by WST-1 assay. Cell proliferation curves for AGS and NCI-N87 cells at indicated times. Error bars represent mean \pm standard deviation (SD) of triplicate experiments. (B) A clonogenic assay was performed on AGS and NCI-N87 cells for 21 days. Left panel shows representative images, and right panel shows quantification of colonies. Error bars represent mean \pm standard deviation of triplicate experiments. ${ }^{* *} \mathrm{p}<0.001$. 
AGS

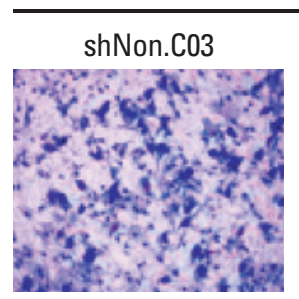

ShER01L.C02

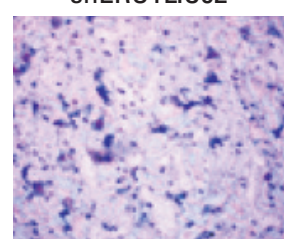

shNon.C06

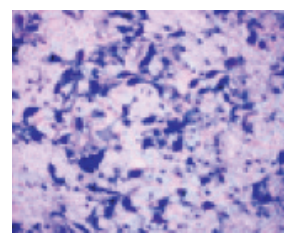

ShER01L.C06

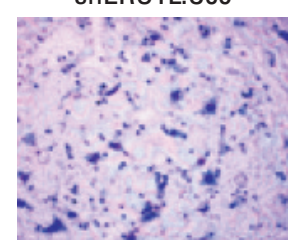

$\mathrm{NCl}-\mathrm{N} 87$

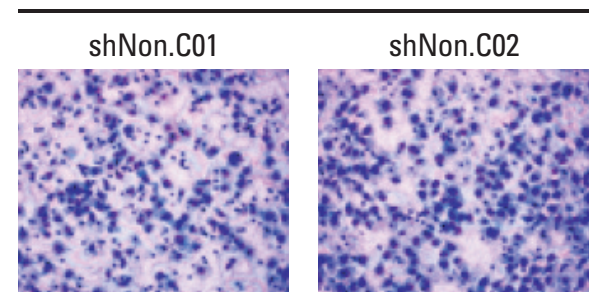

ShER01L.CO4

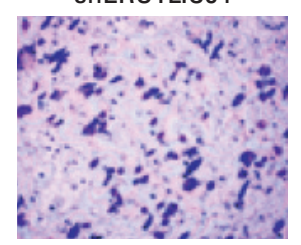

A
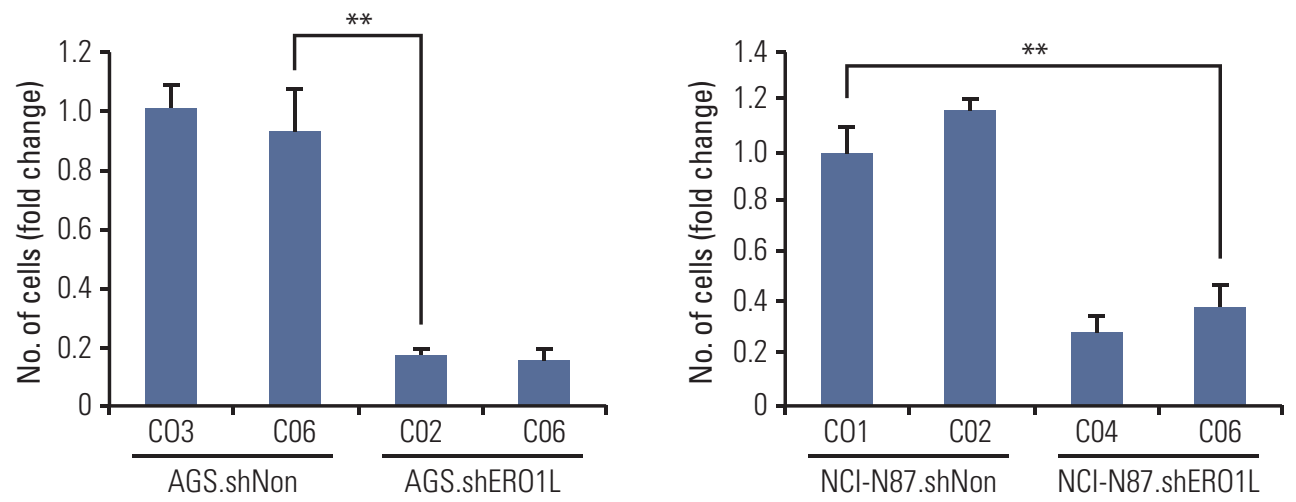

Fig. 8. Silencing of endoplasmic reticulum oxidoreduction 1- $\alpha$ (ERO1L) expression inhibits migration and invasion abilities of AGS and NCI-N87 cells. (A) Cell migration was determined using Boyden chambers. (Continued to the next page)

For this, because directional migration is an important prerequisite of cancer cell invasion, we evaluated the ability of ERO1L expression cells to migrate. Using Boyden chambers, we measured migration of ERO1L-deficient gastric cancer cells (AGS, shERO1L.C02 and C06; NCI-N87, shERO1L.C04 and C06). ERO1L shRNA-mediated knockdown significantly reduced numbers of migratory cells $(\mathrm{p}<0.01$ ) (Fig. 8A). To examine whether or not reduction of cell migration could potentially inhibit invasiveness of these cells, invasion of ERO1L-deficient gastric cancer cells (AGS, shERO1L.C02 and C06; NCI-N87, shERO1L.C04 and C06) into the matrigel membrane was measured by Boyden chamber assay. ERO1L shRNA-mediated knockdown significantly reduced the numbers of invasive cells ( $p<0.001$ ) (Fig. 8B). Intriguingly, these data indicate that ERO1L shRNA-mediated knockdown reduced migration and invasion of AGS and NCI-N87 cells (AGS, shERO1L.C02 and C06; NCI-N87, shERO1L.C04 and C06) compared with the control (AGS, shNon.C03 and C06; NCI-N87, shNon.C01 and C02).

\section{ERO1L is related to chemoresistance in gastric cancer}

We further examined whether or not ERO1L plays a role in chemoresistance in gastric cancer cells. After silencing ERO1L expression with specific shRNA, cell viability assays were performed in gastric cancer cells using a panel of chemotherapy agents, which were commonly used in treatment of gastric cancer, including an anti-metabolite (5-FU) and a microtubule stabilizer (paclitaxel). Interestingly, when ERO1L expression was silenced, AGS and NCI-N87 cells (AGS, shERO1L.C02 and C06; NCI-N87, shERO1L.C04 and C06) became more sensitive to 5-FU and paclitaxel, suggest- 
$\mathrm{NCl}-\mathrm{N} 87$

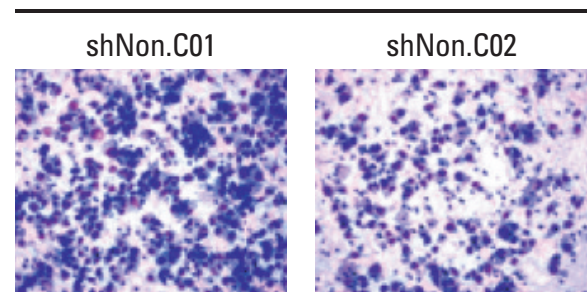

ShER01L.C04

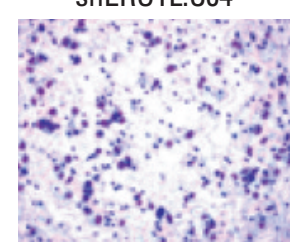

B
AGS

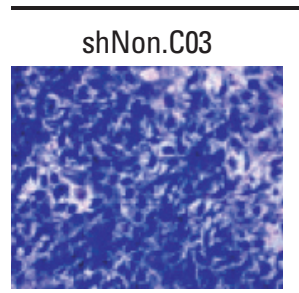

ShER01L.C02

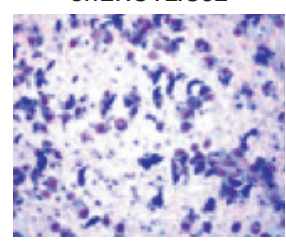

ShER01L.C06

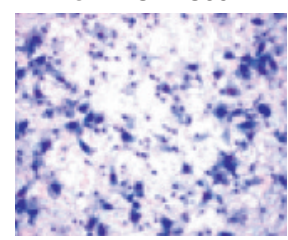

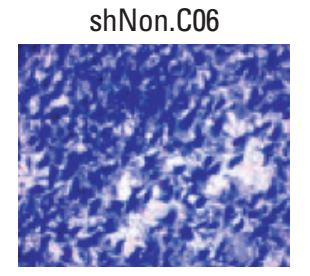

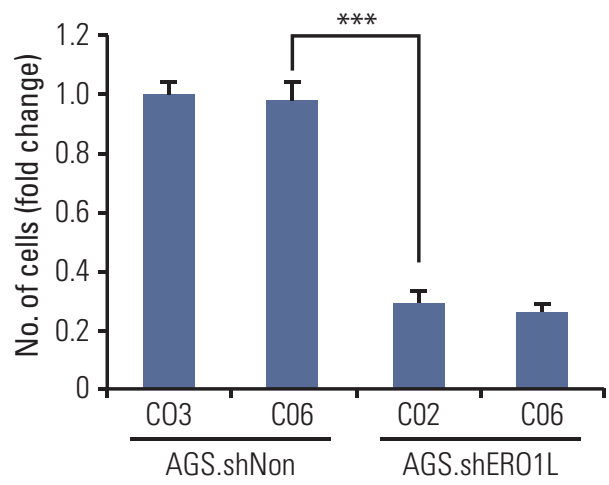

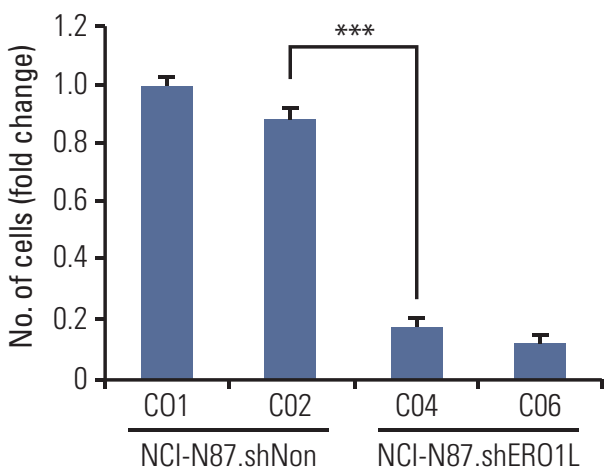

Fig. 8. (Continued from the previous page) (B) Cell invasion was assayed using a membrane coated with Matrigel. Five random microscopic fields were counted for each group. The results presented are an average of five random microscopic fields from three independent experiments. ${ }^{* *} \mathrm{p}<0.01,{ }^{* * *} \mathrm{p}<0.001$.

ing that ERO1L might account for the resistance of gastric cancer cells to 5-FU and paclitaxel (Fig. 9).

\section{Down-regulation of AKT and JNK signaling by ERO1L knockdown}

Several pathways were investigated in order to elucidate the molecular mechanism of ERO1L knockdown-mediated inhibition of gastric cancer proliferation, migration, invasion, and chemoresistance. Several important proteins were differentially expressed between ERO1L-deficient gastric cancer cells (AGS, shERO1L.C02 and C06; NCI-N87, shERO1L.C04 and C06) and shNonTarget vector-infected control cells (AGS, shNon.C03 and C06; NCI-N87, shNon.C01 and C02).

Akt phosphorylation at Ser473 and JNK phosphorylation at Thr183/Tyr185 were surprisingly reduced in ERO1L- deficient gastric cancer cells (AGS, shERO1L.C02 and C06; NCI-N87, shERO1L.C04 and C06) (Fig. 10).

\section{Discussion}

We first obtained ERO1L expression data from tumor tissue samples using qRT-PCR and immunohistochemical assay. Stage III patients who underwent curative gastrectomy were significantly different from patients showing different ERO1L expression levels, as determined by qRT-PCR (Fig. 3A). Similar results were obtained from immunohistochemical assay analysis of gastric cancer specimens (Fig. 3B). Gastric cancer patients showing high ERO1L expression 

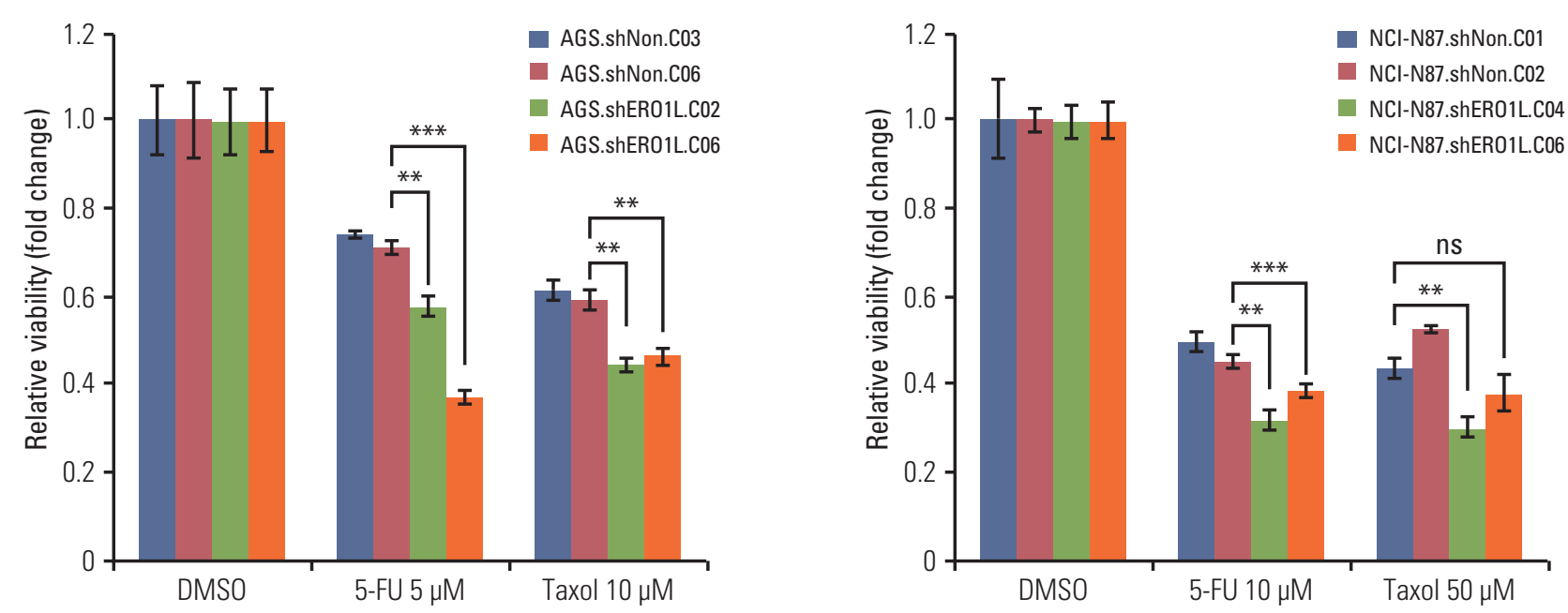

Fig. 9. Endoplasmic reticulum oxidoreduction 1- $\alpha$ (ERO1L)-deficient gastric cancer cells reduce chemoresistance. Viability of ERO1Ldeficient gastric cancer cells treated for 48 hours with chemotherapeutic agents (paclitaxel and 5-fluorouracil [5-FU]) measured by WST-1 assay. Cells treated with dimethylsulfoxide (DMSO) as a control. Data are shown as relative viability (fold change) to the control groups. Data are mean \pm standard deviation of triplicate experiments. ${ }^{* *} \mathrm{p}<0.01$, ${ }^{* * *} \mathrm{p}<0.001$; ns, not significant.

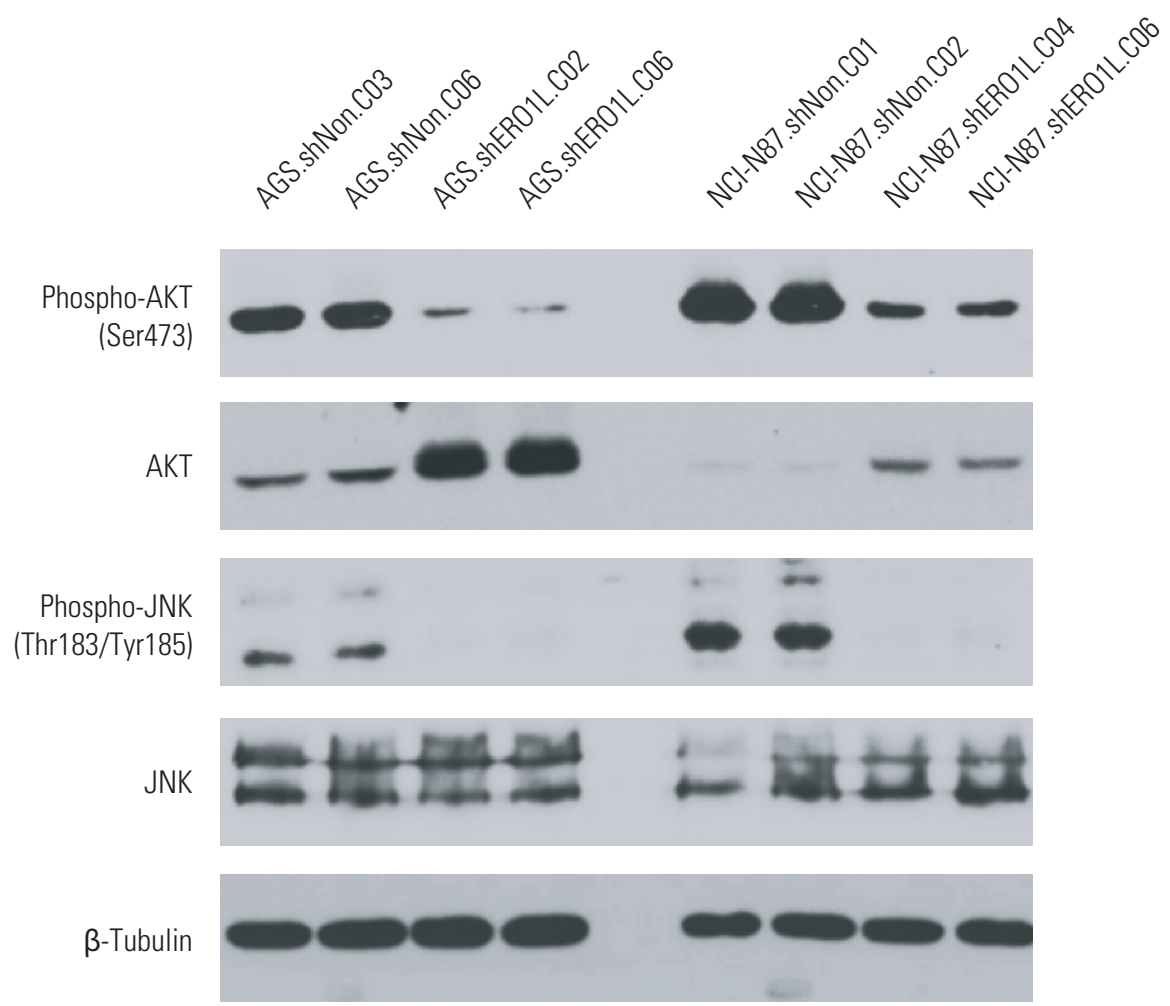

Fig. 10. Inhibitory effects of endoplasmic reticulum oxidoreduction 1- $\alpha$ (ERO1L)-deficient gastric cancer cells on Akt and JNK signaling pathway. Expression levels of total Akt, phosphorylated Akt at Ser473, total JNK, phosphorylated JNK at Thr183/Tyr185, and $\beta$-tubulin (internal control) were measured in the cell lysates. Akt phosphorylation at Ser473 and JNK phosphorylation at Thr183/Tyr185 surprisingly decreased in ERO1L-deficient gastric cancer cells (AGS, shERO1L.C02 and C06; NCI-N87, shERO1L.C04 and C06). 
received a definitively poor prognosis. Thus, intensive treatment and planning of post-adjuvant treatment is required for these patients.

ERO1L plays a pivotal role in VEGF production through disulfide bond formation by protein disulfide isomerase within VEGF protein, suggesting that ERO1L affects tumor growth through angiogenic signaling pathways. Expression of ERO1L is significantly associated with an aggressive breast cancer phenotype, suggesting the potential of ERO1L as a new prognostic marker for breast cancer after surgery. However, how ERO1L expression is regulated under hypoxia and within tumor cells should be determined. More importantly, the precise mechanism of tumor cell growth should be elucidated.

In this study, we demonstrated that ERO1L-deficient gastric cancer cells (AGS, shERO1L.C02 and C06; NCI-N87, shERO1L.C04 and C06) could effectively suppress cell proliferation and inhibit colony formation, migration, invasiveness, and chemoresistance (Figs. 7-9). According to our results, ERO1L is an attractive therapeutic target for the treatment of gastric cancer. The current results demonstrate that inhibition of the phosphoinositide 3-kinase (PI3K) and JNK mitogen-activated protein kinase (MAPK) signaling pathways plays an important role in cell cycle transition, proliferation, and metastasis. Furthermore, dual inhibition of the PI3K and MAPK pathways has synergy in lung cancers that are unresponsive to single PI3K inhibition. Akt (also called $\mathrm{PKB}$ ) is an important downstream effector of PI3K, and activated Akt translocates to the cytoplasm and nucleus to activate downstream targets involved in survival, proliferation, cell cycle progression, migration, and angiogenesis [19]. Phosphorylation of Akt at Ser473 is increased in poorly differentiated prostate cancer cells [20] and is a good predictor of poor clinical outcome in cancer patients [21]. The stressactivated protein kinase pathway, JNK, alternatively referred to as SAPK/JNK, also plays an important role in control of cell proliferation in a wide variety of cell types. Reduced activation of JNK could be beneficial in controlling growth in tumors expressing over-activated JNK [22]. Activated JNK may act as an oncoprotein due to its ability to activate the transcription factor component c-JUN or inactivate the proapoptotic protein BAD [23]. Thus, reduced activation of JNK could be beneficial for controlling growth of tumors expressing over-activated JNK. Together, these results indicate that
ERO1L expression could potentially modulate activity of the Akt and JNK pathway in gastric cancer cells.

The results of our study show that ERO1L-deficient gastric cancer cells (AGS, shERO1L.C02 and C06; NCI-N87, shERO1L.C04 and C06) led to reduction of Akt phosphorylation at Ser473, as expected, due to reduction of Akt activation. Likewise, shRNA-mediated ERO1L knockdown led to decreased phosphorylation of JNK at Thr183/Tyr185. Taken together, inhibition of the Akt and JNK pathways by shRNAmediated ERO1L knockdown in gastric cancer cells points to a potential oncogenic role for ERO1L in gastric cancer.

\section{Conclusion}

High expression of ERO1L is implicated in gastric cancer, particularly in tumors with poor-prognostic features. ERO1L is a promising prognostic marker of gastric cancer, and personalized adjuvant treatment based on ERO1L expression levels would be an effective practice for treatment of gastric cancer. Administration of the appropriate drug improves clinical outcomes and decreases toxicity.

\section{Conflicts of Interest}

Conflict of interest relevant to this article was not reported.

\section{Acknowledgments}

This work was supported by the Basic Science Research Program through the National Research Foundation of Korea (NRF) funded by the Ministry of Education, Science and Technology (MEST) (NRF-2010-0024248) and a CMB-Yuhan research grant of Yonsei University College of Medicine (6-2013-0065).

\section{References}

1. Jemal A, Bray F, Center MM, Ferlay J, Ward E, Forman D. Global cancer statistics. CA Cancer J Clin. 2011;61:69-90.
2. Lynch HT, Grady W, Suriano G, Huntsman D. Gastric cancer: new genetic developments. J Surg Oncol. 2005;90:114-33. 
3. Shah MA, Ajani JA. Gastric cancer: an enigmatic and heterogeneous disease. JAMA. 2010;303:1753-4.

4. Gallo A, Cha C. Updates on esophageal and gastric cancers. World J Gastroenterol. 2006;12:3237-42.

5. Bang YJ, Kim YW, Yang HK, Chung HC, Park YK, Lee KH, et al. Adjuvant capecitabine and oxaliplatin for gastric cancer after D2 gastrectomy (CLASSIC): a phase 3 open-label, randomised controlled trial. Lancet. 2012;379:315-21.

6. Tay ST, Leong SH, Yu K, Aggarwal A, Tan SY, Lee CH, et al. A combined comparative genomic hybridization and expression microarray analysis of gastric cancer reveals novel molecular subtypes. Cancer Res. 2003;63:3309-16.

7. Kim B, Bang S, Lee S, Kim S, Jung Y, Lee C, et al. Expression profiling and subtype-specific expression of stomach cancer. Cancer Res. 2003;63:8248-55.

8. Chen X, Leung SY, Yuen ST, Chu KM, Ji J, Li R, et al. Variation in gene expression patterns in human gastric cancers. Mol Biol Cell. 2003;14:3208-15.

9. Lee HS, Cho SB, Lee HE, Kim MA, Kim JH, Park DJ, et al. Protein expression profiling and molecular classification of gastric cancer by the tissue array method. Clin Cancer Res. 2007;13: 4154-63.

10. Tan IB, Ivanova T, Lim KH, Ong CW, Deng N, Lee J, et al. Intrinsic subtypes of gastric cancer, based on gene expression pattern, predict survival and respond differently to chemotherapy. Gastroenterology. 2011;141:476-85.

11. Ooi CH, Ivanova T, Wu J, Lee M, Tan IB, Tao J, et al. Oncogenic pathway combinations predict clinical prognosis in gastric cancer. PLoS Genet. 2009;5:e1000676.

12. Cho JY, Lim JY, Cheong JH, Park YY, Yoon SL, Kim SM, et al. Gene expression signature-based prognostic risk score in gastric cancer. Clin Cancer Res. 2011;17:1850-7.

13. Bristow RG, Hill RP. Hypoxia and metabolism: hypoxia, DNA repair and genetic instability. Nat Rev Cancer. 2008;8:180-92.

14. Benham AM, Cabibbo A, Fassio A, Bulleid N, Sitia R, Braak- man I. The CXXCXXC motif determines the folding, structure and stability of human Ero1-Lalpha. EMBO J. 2000;19: 4493-502.

15. Gess B, Hofbauer KH, Wenger RH, Lohaus C, Meyer HE, Kurtz A. The cellular oxygen tension regulates expression of the endoplasmic oxidoreductase ERO1-Lalpha. Eur J Biochem. 2003;270:2228-35.

16. May D, Itin A, Gal O, Kalinski H, Feinstein E, Keshet E. Ero1L alpha plays a key role in a HIF-1-mediated pathway to improve disulfide bond formation and VEGF secretion under hypoxia: implication for cancer. Oncogene. 2005;24:1011-20.

17. Simon R, Lam A, Li MC, Ngan M, Menenzes S, Zhao Y. Analysis of gene expression data using BRB-ArrayTools. Cancer Inform. 2007;3:11-7.

18. Eisen MB, Spellman PT, Brown PO, Botstein D. Cluster analysis and display of genome-wide expression patterns. Proc Natl Acad Sci U S A. 1998;95:14863-8.

19. Bellacosa A, Kumar CC, Di Cristofano A, Testa JR. Activation of AKT kinases in cancer: implications for therapeutic targeting. Adv Cancer Res. 2005;94:29-86.

20. Malik SN, Brattain M, Ghosh PM, Troyer DA, Prihoda T, Bedolla R, et al. Immunohistochemical demonstration of phospho-Akt in high Gleason grade prostate cancer. Clin Cancer Res. 2002;8:1168-71.

21. Kreisberg JI, Malik SN, Prihoda TJ, Bedolla RG, Troyer DA, Kreisberg S, et al. Phosphorylation of Akt (Ser473) is an excellent predictor of poor clinical outcome in prostate cancer. Cancer Res. 2004;64:5232-6.

22. Ali A, Sidorova TS, Matesic DF. Dual modulation of JNK and Akt signaling pathways by chaetoglobosin $\mathrm{K}$ in human lung carcinoma and ras-transformed epithelial cells. Invest New Drugs. 2013;31:525-34.

23. Engelberg D. Stress-activated protein kinases-tumor suppressors or tumor initiators? Semin Cancer Biol. 2004;14:271-82. 